\title{
Pancreatic tumours derive amino acids via extracellular protein uptake
}

Spontaneously arising pancreatic tumours in mice source amino acids from the extracellular environment, according to new research published in the journal Nature Medicine. Using microdevices implanted into pancreatic tumours, the researchers show that local inhibition of macropinocytosis reduces amino acid levels in tumour cells but not adjacent healthy tissue.

Altered cellular metabolism has a well-appreciated involvement in tumorigenesis, enabling cancer cells to derive adequate nutrients to remain viable and proliferate. Macropinocytosis, a process by which extracellular soluble material is internalized in bulk, is known to contribute to the provision of protein in pancreatic cancer cells in vitro, yet the importance of this pathway in vivo has been less well studied.

"In order to trace the fate of amino acids in protein in tissues, we needed a way to deliver isotope-labelled amino acids to tissues in mice," explains author Matthew Vander Heiden. In wild-type and transgenic mice spontaneously developing pancreatic ductal adenocarcinoma (PDAC), the investigators exchanged endogenous plasma albumin for ${ }^{15} \mathrm{~N}$-labelled albumin using plasmaphoresis. "By exchanging several blood volumes we could obtain sufficient enrichment of labelled albumin to track its fate in tissues," states Vander Heiden. Tumour-bearing mice had increased levels of labelled amino acids $12 \mathrm{~h}$ after plasmaphoresis, indicating increased protein catabolism; in addition, pancreatic tumours displayed enrichment of labelled amino acids compared with healthy pancreatic tissue.

Next, a microdevice to deliver boron-dipyrromethene-labelled albumin, enabling visualization of albumin degradation, was implanted within the tumours. After $24 \mathrm{~h}$, tumour tissue adjacent to the implants was positive for albumin catabolism, whereas no signal was observed when implants were located next to non-tumour tissue. Labelled fibronectin - a component of extracellular matrix, which is a primary constituent of stroma - delivered by the microdevice was found to be taken up by tumours but not by normal pancreatic tissue, further providing evidence for extracellular protein consumption by PDAC tumours in vivo.

To assess whether inhibiting macropinocytosis might alter uptake of extracellular protein and tumour amino acid levels, Vander Heiden and colleagues implanted microdevices containing

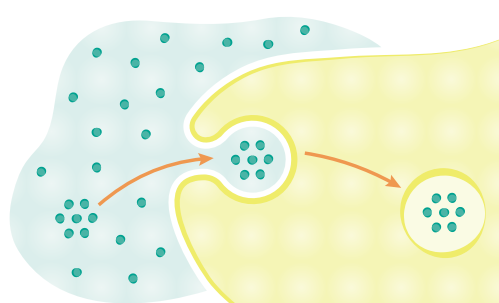

ethylisopropylamiloride (EIPA) or inert vehicle into PDAC tumours in their mouse model. Using imaging mass spectrometry on tissue slices, the researchers demonstrated lower concentrations of the amino acids glutamate, aspartate, glutamine and histidine in tumour tissue exposed to EIPA than in adjacent, non-exposed tissue.

Vander Heiden is hopeful that the methods employed in this study can be applied to assess protein metabolism in other solid tumours. "We remain very interested in which other tumours also rely on macropinocytosis to obtain amino acids, and more broadly defining the different fuels cancers use and the factors determining differential nutrient utilization," he concludes.

Hugh Thomas

ORIGINAL ARTICLE Davidson, S. M. et al. Direct evidence for cancer-cell-autonomous extracellular protein catabolism in pancreatic tumors. Nat. Med. http://dx.doi.org/10.1038/ nm.4256 (2016) 